\title{
Family history of type 1 and type 2 diabetes and risk of latent autoimmune diabetes in adults (LADA)
}

\author{
Hjort, R.
}

2017-12

Hjort , R , Alfredsson , L , Andersson , T , Carlsson , P -O , Grill , V , Groop , L , Martinell , M , Rasouli , B , Storm , P , Tuomi , T \& Carlsson , S 2017 , ' Family history of type 1 and type 2 diabetes and risk of latent autoimmune diabetes in adults (LADA) ', Diabetes \& metabolism , vol. 43 , no. 6 , pp. 536-542 . https://doi.org/10.1016/j.diabet.2017.05.010

http://hdl.handle.net/10138/298240

https://doi.org/10.1016/j.diabet.2017.05.010

publishedVersion

Downloaded from Helda, University of Helsinki institutional repository.

This is an electronic reprint of the original article.

This reprint may differ from the original in pagination and typographic detail.

Please cite the original version. 


\title{
Original article
}

\section{Family history of type 1 and type 2 diabetes and risk of latent autoimmune diabetes in adults (LADA)}

\author{
R. Hjort ${ }^{\mathrm{a}, *}$, L. Alfredsson ${ }^{\mathrm{b}}$, T. Andersson ${ }^{\mathrm{a}, \mathrm{c}}$, P.-O. Carlsson ${ }^{\mathrm{d}}$, V. Grill ${ }^{\mathrm{e}, \mathrm{f}}$, L. Groop ${ }^{\mathrm{g}}$,

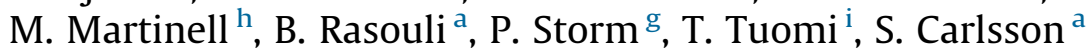 \\ ${ }^{a}$ Unit of epidemiology, institute of environmental medicine, Karolinska Institutet, Stockholm, Sweden \\ ${ }^{\mathrm{b}}$ Unit of cardiovascular epidemiology, institute of environmental medicine, Karolinska Institutet, Stockholm, Sweden \\ ${ }^{\mathrm{c}}$ Centre for occupational and environmental medicine, Stockholm County Council, Sweden \\ 'Department of medical sciences, Uppsala university, Uppsala, Sweden \\ ${ }^{\mathrm{e}}$ NTNU institute of cancer research and molecular medicine, Norwegian university of science and technology, Trondheim, Norway \\ ${ }^{\mathrm{f}}$ Department of endocrinology, Trondheim university hospital, Trondheim, Norway \\ $\mathrm{g}$ Department of clinical sciences in Malmö, clinical research centre, Lund university, Malmö, Sweden \\ ${ }^{\mathrm{h}}$ Department of public health and caring sciences, Uppsala university, Uppsala, Sweden \\ ${ }^{\mathrm{i}}$ Division of endocrinology, abdominal centre, Finnish institute for molecular medicine and research program for diabetes and obesity, university of Helsinki \\ and Folkhälsan research centre, Helsinki university hospital, Helsinki, Finland
}

\section{A R T I C L E I N F O}

\section{Article history}

Received 17 January 2017

Received in revised form 11 May 2017

Accepted 23 May 2017

Available online 29 June 2017

\section{Keywords:}

Autoimmune diabetes

Case-control study

Family history of diabetes

Heredity

Latent autoimmune diabetes in adults

Type 2 diabetes

\begin{abstract}
A B S T R A C T
Background. - A family history of diabetes (FHD) is a strong predictor of diabetes risk, yet has rarely been investigated in latent autoimmune diabetes in adults (LADA). This study therefore investigated the risk of LADA and type 2 diabetes (T2D) in relation to FHD, taking into account the type of diabetes in relatives. Methods. - Data from a population-based study were used, including incident cases of LADA [glutamic acid decarboxylase antibody (GADA)-positive, $n=378$ ] and T2D (GADA-negative, $n=1199$ ), and their matched controls $(n=1484)$. First-degree relatives with disease onset at age $<40$ years and taking insulin treatment were classified as type 1 diabetes (T1D) or, if otherwise, as T2D. Odds ratios (ORs) were adjusted for age, gender, BMI, education and smoking. Cases were genotyped for high- and low-risk HLA genotypes.

Results. - Both FHD-T1D (OR: 5.8; 95\% CI: 3.2-10.3) and FHD-T2D (OR: 1.9; 95\% CI: 1.5-2.5) were associated with an increased risk of LADA, whereas the risk of T2D was associated with FHD-T2D (OR: 2.7; 95\% CI: 2.2-3.3), but not FHD-T1D. In LADA patients, FHD-T1D vs FHD-T2D was associated with higher GADA but lower C-peptide levels, lower prevalence of low-risk HLA genotypes (5.0\% vs $28.6 \%$, respectively; $P=0.038)$ and a tendency for higher prevalence of high-risk genotypes $(90.0 \%$ vs $69.1 \%$, respectively; $P=0.0576$ ).

Conclusion. - The risk of LADA is substantially increased with FHD-T1D but also, albeit significantly less so, with FHD-T2D. This supports the idea of LADA as a mix of both T1D and T2D, but suggests that the genes related to T1D have greater impact. LADA patients with FHD-T1D had more T1D-like features, emphasizing the heterogeneity of LADA.
\end{abstract}

(c) 2017 Elsevier Masson SAS. All rights reserved.

\footnotetext{
Abbreviations: ANDIS, All New Diabetics in Scania; ANDiU, All New Diabetics in Uppsala; ESTRID, Epidemiological Study of Risk Factors for LADA and Type 2 Diabetes; FHD-T1D, Family history of type 1 diabetes; FHD-T2D, Family history of type 2 diabetes; GADA, Glutamic acid decarboxylase antibody; HLA, Human leucocyte antigen; LADA, Latent autoimmune diabetes in adults.

* Corresponding author at: Unit of epidemiology, institute of environmental medicine, Karolinska Institutet, 17177 Stockholm, Sweden.

E-mail address: rebecka.hjort@ki.se (R. Hjort).
}

\section{Introduction}

A family history of diabetes (FHD) encompasses both genetic and shared environmental factors, and is a strong predictor of diabetes risk. Previous studies have indicated that diabetes in firstdegree relatives confers a ninefold greater risk of type 1 diabetes (T1D) [1] and a threefold greater risk of type 2 diabetes (T2D) [2]. The genetic risk of T1D is mainly attributed to genes in the human leucocyte antigen (HLA) region, which are estimated to 
explain around $30-50 \%$ of familial clusterings [3]. Recent studies have identified several genes related to T2D, of which variants within the TCF7L2 gene have the strongest effect [4]. The currently identified genes, however, can only explain about $20 \%$ of the genetic heritability of T2D [5] and only a fraction of its familial aggregation [2]. Therefore, FHD remains an important, easily obtained predictor of diabetes risk [2].

Latent autoimmune diabetes in adults (LADA) is thought to be a genetic mix of both T1D and T2D [6], and linked to HLA DQB1 risk genotypes associated with autoimmunity $[7,8]$ as well as genes associated with T2D, including TCF7L2 [9-11]. Consequently, a family history of both type 1 diabetes (FHD-T1D) and type 2 diabetes (FHD-T2D) may promote LADA. Findings from prospective data indicate that FHD increases LADA risk two- to fourfold [12,13], while cross-sectional studies also reveal a high prevalence of FHD $[14,15]$. While studies of the relative importance of FHD-T1D vs FHD-T2D are scarce, data from a few small-scale studies support links to both T1D and T2D [16-20]. Also, in a prospective follow-up study of a non-diabetic population, FHDT1D conferred a twofold greater risk of developing non-insulindependent diabetes [13].

Our aim was to clarify the role of FHD-T1D and FHD-T2D in relation to LADA and T2D. To this end, data were taken from a novel Swedish population-based study to create the largest study to date of FHD and LADA.

\section{Methods}

\section{Study population and design}

The present study used data from the Epidemiological Study of Risk Factors for LADA and Type 2 Diabetes (ESTRID), a populationbased study including incident cases. A detailed description of the study design has been previously reported [21]. Briefly, ESTRID is a substudy of ANDIS (All New Diabetics in Scania) [22], a detailed study aiming to characterize all new cases of diabetes in the Swedish county of Scania. For ESTRID, all newly diagnosed LADA patients and a random sample of newly diagnosed T2D cases (four per each LADA case) were invited to participate from 2010 onwards. For each case of LADA, six diabetes-free controls, aged $\geq 35$ years (to match the definition of LADA), were randomly selected from the general population of Scania, and matched by time and region (incidence density sampling) [23]. After 2012, cases and controls were also recruited from Uppsala county through ANDiU (All New Diabetics in Uppsala) [24], a sister study to ANDIS. Response rate was high: $80 \%$ vs $64 \%$ for cases vs controls, respectively. Thus, the analytical sample of the present study included 378 cases of LADA, 1199 cases of T2D and 1484 controlsall of the participants included in ESTRID up to 31 July 2015-with complete information on their age, gender, FHD, body mass index (BMI), education and smoking (99.5\% of the total sample).

The study was approved by the ethics review board in Stockholm (reference number: 2010/336-31/2), and all participants provided their written informed consent.

\section{Biochemical analysis and case definition}

At diagnosis, all patients provided blood samples, which were analyzed for glutamic acid decarboxylase antibody (GADA), Cpeptide and glucose at the central laboratories of the university hospitals of Scania and Uppsala. GADA was analyzed in serum by enzyme-linked immunosorbent assay (ELISA; RSR Ltd, Cardiff, UK). Levels $\geq 10 \mathrm{IU} / \mathrm{mL}$ were regarded as positive, as per instructions in the kit. At a cut-off level of $10.7 \mathrm{IU} / \mathrm{mL}$, sensitivity was $84 \%$ and specificity 98\% [25]. Fasting (overnight) levels of C-peptide in plasma were measured by an IMMULITE 2000 (Siemens Healthcare Diagnostics Product Ltd., Caernarfon, UK) or Cobas e 601 (Roche Diagnostics, Mannheim, Germany) analyzer [26]. LADA patients had onset at age $\geq 35$ years, were GADA-positive ( $\geq 10 \mathrm{IU} / \mathrm{mL}$ ) and had C-peptide levels above the lower limit of normal range [ $\geq 0.2 \mathrm{nmol} / \mathrm{L}$ (IMMULITE) or $\geq 0.3 \mathrm{nmol} / \mathrm{L}$ (Cobas e 601)]. T2D patients had onset at age $\geq 35$ years, were GADA-negative and had C-peptide levels $>0.6 \mathrm{nmol} / \mathrm{L}$ (IMMULITE) or $>0.72 \mathrm{nmol} / \mathrm{L}$ (Cobas e 601). While there is no established definition of LADA, this is in line with previously used criteria [6] except for C-peptide, which was used as an indicator of any remaining insulin production. This criterion distinguished LADA patients from those with adult-onset T1D (C-peptide $<0.2$ or $0.3 \mathrm{nmol} / \mathrm{L}$ ), who were not recruited into ESTRID. C-peptide may be regarded as a more objective measurement than the more commonly used insulin criteria [27].

Homoeostasis model assessment (HOMA) was used to assess insulin resistance (HOMA-IR), insulin sensitivity (HOMA-S) and $\beta$ cell function (HOMA- $\beta$ ), based on the relationship between fasting glucose and C-peptide [28]. DNA was extracted and genetic analyses were performed at the central laboratory in Scania by running the iPLEX Gold Assay (Sequenom Inc, San Diego, CA, USA). As per the previously described methodology [29], three singlenucleotide polymorphisms (SNPs) in the MHC region (rs3104413, rs2854275, rs9273363) were combined to identify carriers of the high- and low-risk HLA $D R$ and $D Q$ genotypes known to be associated with autoimmune diabetes (DR3/4, DR3/3, DR4/4, DR3/X, $D R 4 / X, D R 4-D Q 7, D R 4 / 3-D Q 8, D R 4-D Q 8, D R X / X)$, where the greatest risk of T1D is found in subjects heterozygous for these types $[29,30]$. Genetic information was available for $70.1 \%$ of LADA cases and $68.6 \%$ of T2D cases. No genetic and clinical information was available for the controls.

\section{Questionnaire}

Cases and controls answered an extensive questionnaire with items on heredity, previous health, and lifestyle and demographic socioeconomic and psychosocial factors. Cases were given the questionnaire following diagnosis (within a median time of 6 months), and were carefully instructed to report conditions as they were prior to diagnosis.

\section{Family history of diabetes}

Information on FHD entailed questions on diabetes in firstdegree relatives (mother, father, sisters, brothers, daughters, sons) as well as in maternal and paternal grandparents. Also, the questionnaire asked about the total number of other, seconddegree relatives (grandchildren, aunts, uncles, nephews, nieces) and first cousins with diabetes. (This report included first cousins as second-degree relatives as they could not be distinguished from other relatives.) For first-degree relatives, information on age at diagnosis and modalities of treatment were also obtained. Relatives were classified as FHD-T1D if they had onset at age $<40$ years and were taking insulin treatment or, if otherwise, as FHD-T2D.

\section{Covariates}

Self-reported information on current body weight together with height was used to calculate BMI $\left(\mathrm{kg} / \mathrm{m}^{2}\right)$. For these patients, this information correlated significantly with information from medical records $(r=0.92)$. Information on smoking was obtained through questions about current and previous tobacco use, and included duration and average number of cigarettes smoked per day. Physical activity was assessed by validated questions about leisure-time physical activity during the preceding year [31]. 
Information on average alcohol intake during the preceding year was used to calculate average daily consumption of alcohol (g/ day). Participants were also asked to report their highest level of education.

\section{Statistical analysis}

Baseline characteristics of participants are presented as proportions, means or medians together with two-sided $P$-values, calculated by $\chi^{2}$ (proportions), Student's $t$ (means) and KruskalWallis (medians) tests. Odds ratios (ORs) of diabetes by FHD and 95\% confidence intervals (CIs) were calculated by conditional logistic regression. As the study participants were recruited through incidence density sampling [23], the ORs are interpreted as incidence rate ratios. All analyses were controlled for age and gender (model 1) and, in addition, for BMI $\left(\mathrm{kg} / \mathrm{m}^{2}\right)$, smoking (never, former, current) and education (primary school, upper secondary school, university; model 2). Results from model 2 are presented in text and tables, unless otherwise specified. Further adjustments for physical activity and alcohol consumption had only a minor influence on ORs and were therefore not included in the final model. Analyses of FHD-T1D were adjusted for FHD-T2D and vice versa. Stratified analyses were also performed by median GADA levels, with LADA $_{\text {low }}$ defined as $<177.5 \mathrm{IU} / \mathrm{mL}$ and LADA $_{\text {high }}$ as $\geq 177.5 \mathrm{IU} / \mathrm{mL}$. Sensitivity analyses used a more stringent criterion to classify FHD-T2D ( $\geq 40$ years and no insulin treatment). Statistical Analysis Software (SAS) version 9.4 (SAS Institute, Cary, NC, USA) was used for all statistical analyses.

\section{Results}

\section{Characteristics}

As expected, patients with LADA were younger at onset, leaner, had lower levels of C-peptide and HOMA- $\beta$, and were more often treated with insulin than patients with T2D (43.5\% vs $5.9 \%$, $P<0.0001$; Table 1). FHD-T1D was reported by $10.1 \%$ and FHDT2D by $35.5 \%$ of patients with LADA. Corresponding frequencies in those with T2D were $4.8 \%$ and $46.2 \%$, respectively. Of the genotype combinations investigated, the most common were high-risk DR4$D Q 8$ and low-risk $D R X / X$ (Table SI; see supplementary materials associated with this article online). LADA patients were more likely than T2D patients to carry the DR4-DQ8 high-risk genotype and less likely to carry the $D R X / X$ low-risk genotype. On combining all high-risk genotypes (DR4-DQ8, $D R 3 / 3, D R 4 / 4, D R 3 / 4, D R 3 / X, D R 4 / X)$ [29], the prevalence for LADA vs T2D was $72.1 \%$ vs $46.2 \%$, respectively $(P<0.0001)$. A larger proportion of patients (including both LADA and T2D) who reported FHD-T1D had the high-risk genotype (59.0\% vs 31.03\%; $P=0.0006$ ) and a lower proportion had the low-risk genotype $(12.8 \%$ vs $44.4 \% ; P<0.0001)$ compared with LADA and T2D patients reporting FHD-T2D.

\section{Family history of diabetes, LADA and type 2 diabetes}

Diabetes in either first- or second-degree relatives conferred a twofold greater risk of LADA (Table 2), and the risk increased with the number and closeness of the relatives. This risk was increased irrespective of whether a parent, sibling or child had diabetes. The impact of having a first- vs second-degree relative with diabetes was similar for T2D, whereas the risk appeared to be particularly pronounced in those with an affected sibling (OR: 2.9, 95\% CI: 2.23.9). Adjusting for BMI, smoking and education had only a minor influence on these associations (Table 2).

\section{Family history of type 1 and type 2 diabetes}

Separate analyses by diabetes type (Fig. 1) indicated that the risk of LADA was significantly more pronounced for FHD-T1D (OR: 5.8, 95\% CI: 3.2-10.3) than for FHD-T2D (OR: 1.9, 95\% CI: 1.5-2.5). The excess risk associated with FHD-T1D was seen irrespective of which parent or sibling had T1D, and was similar for male and female relatives. However, a child with T1D conferred no added risk. With regard to FHD-T2D, the increased risk of LADA appeared to be more pronounced if a brother or child had diabetes. Sensitivity analyses indicated that the association between LADA and FHD-T2D was similar (OR: 1.8, 95\% CI: 1.32.6) when a stricter classification of FHD-T2D (onset at age $\geq 40$ years and no insulin treatment) was used. Stratification by GADA levels indicated that the association with FHD-T1D was stronger for LADA $\mathrm{L}_{\text {high }}$ (OR: 6.9, 95\% CI: 3.5-13.4) than for $\mathrm{LADA}_{\text {low }}$ (OR: 4.9, 95\% CI: 2.2-11.2), whereas the association with FHDT2D was similar for both LADA $_{\text {high }}$ (OR: $1.8,95 \% \mathrm{CI}: 1.2-2.5$ ) and LADA $_{\text {low }}$ (OR: 2.0, 95\% CI: 1.4-2.9). In addition, the risk of T2D was

Table 1

Characteristics of cases and controls.

\begin{tabular}{|c|c|c|c|c|}
\hline Characteristics & Controls & $\mathrm{T} 2 \mathrm{D}$ & LADA & $P^{\mathrm{a}}$ \\
\hline Subjects, $n$ & 1484 & 1199 & 378 & - \\
\hline Women, \% & 52.8 & 39.5 & 46.8 & 0.0120 \\
\hline Age, years, mean $(\mathrm{SD})^{\mathrm{b}}$ & $58.5(13.4)$ & $63.1(10.3)$ & $58.7(12.5)$ & $<0.0001$ \\
\hline Body mass index, $\mathrm{kg} / \mathrm{m}^{2}$, mean (SD) & $25.9(4.1)$ & $31.1(5.4)$ & $28.1(5.3)$ & $<0.0001$ \\
\hline First-degree family history of diabetes, \% & 23.8 & 48.2 & 43.4 & 0.1016 \\
\hline Family history of T1D, \% & 2.4 & 4.8 & 10.1 & 0.0002 \\
\hline Family history of T2D, \% & 22.1 & 46.2 & 35.5 & 0.0002 \\
\hline With insulin treatment, \% & - & 5.9 & 43.5 & $<0.0001$ \\
\hline GADA, IU/mL, median (IQR) ${ }^{\mathrm{c}}$ & - & - & $177.5(225.0)$ & - \\
\hline C-peptide, nmol/L, mean $(\mathrm{SD})^{\mathrm{C}}$ & - & $1.33(0.58)$ & $0.81(0.53)$ & $<0.0001$ \\
\hline HOMA-IR, mean $(\mathrm{SD})^{\mathrm{c}}$ & - & $5.79(13.39)$ & $5.22(13.03)$ & 0.5147 \\
\hline HOMA- $\beta$, mean $(S D)^{c}$ & - & $69.56(35.91)$ & $45.21(35.27)$ & $<0.0001$ \\
\hline HOMA-S, mean $(S D)^{c}$ & - & $29.31(12.89)$ & $40.97(24.87)$ & $<0.0001$ \\
\hline HLA $D R 4-D Q 8, \%$ & - & 26.3 & 53.6 & $<0.0001$ \\
\hline High-risk HLA genotypes ${ }^{\mathrm{d}}, \%$ & - & 46.2 & 72.1 & $<0.0001$ \\
\hline HLA $D R X / X, \%$ & - & 47.8 & 24.9 & $<0.0001$ \\
\hline
\end{tabular}

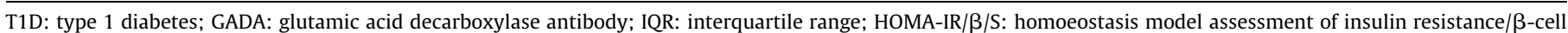

function/insulin sensitivity; HLA: human leucocyte antigen.

a Between LADA (latent autoimmune diabetes in adults) and T2D (type 2 diabetes).

b At onset for cases of LADA and T2D.

c Eight LADA cases and 26 T2D did not consent to use of their clinical information, including C-peptide and GADA measurements.

d $D R 4-D Q 8, D R 3 / 3, D R 4 / 4, D R 3 / 4, D R 3 / X, D R 4 / X$ combined. 
Table 2

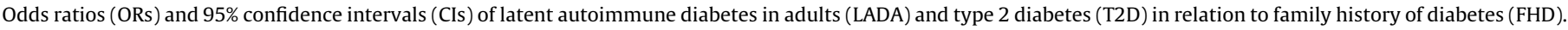

\begin{tabular}{|c|c|c|c|c|c|c|}
\hline & \multicolumn{3}{|l|}{ LADA } & \multicolumn{3}{|l|}{$\mathrm{T} 2 \mathrm{D}$} \\
\hline & Cases/controls & $\mathrm{OR}^{\mathrm{a}}(95 \% \mathrm{CI})$ & $\mathrm{OR}^{\mathrm{b}}(95 \% \mathrm{CI})$ & Cases/controls & $\mathrm{OR}^{\mathrm{a}}(95 \% \mathrm{CI})$ & $\mathrm{OR}^{\mathrm{b}}(95 \% \mathrm{CI})$ \\
\hline No FHD & $154 / 872$ & 1 & 1 & $464 / 872$ & 1 & 1 \\
\hline Any FHD & $224 / 612$ & $2.20(1.73-2.78)$ & $2.08(1.63-2.65)$ & $735 / 612$ & $2.56(2.17-3.03)$ & $2.30(1.89-2.80)$ \\
\hline 1st degree & $164 / 353$ & $2.38(1.87-3.04)$ & $2.22(1.73-2.85)$ & $578 / 353$ & $2.99(2.50-3.57)$ & $2.66(2.17-3.28)$ \\
\hline 2nd degree & $60 / 259$ & $1.45(1.13-1.87)$ & $1.43(1.10-1.85)$ & $157 / 259$ & $1.45(1.20-1.75)$ & $1.48(1.18-1.84)$ \\
\hline \multicolumn{7}{|c|}{ Relatives with diabetes $(n)$} \\
\hline 1 & $95 / 371$ & $1.53(1.15-2.05)$ & $1.48(1.10-1.99)$ & $333 / 371$ & $1.85(1.52-2.26)$ & $1.68(1.33-2.12)$ \\
\hline 2 & $72 / 148$ & $2.93(2.09-4.10)$ & $2.67(1.89-3.78)$ & $182 / 148$ & $2.62(2.01-3.40)$ & $2.26(1.67-3.06)$ \\
\hline$>2$ & $49 / 86$ & $3.38(2.28-5.02)$ & $3.19(2.13-4.78)$ & $209 / 86$ & $5.97(4.42-8.08)$ & $5.33(3.78-7.53)$ \\
\hline Any parent & $115 / 275$ & $1.55(1.18-2.04)$ & $1.48(1.12-1.95)$ & $419 / 275$ & $2.16(1.77-2.63)$ & $1.99(1.58-2.49)$ \\
\hline Mother & $53 / 135$ & $1.48(1.03-2.12)$ & $1.38(0.95-2.00)$ & $204 / 135$ & $2.10(1.61-2.72)$ & $1.88(1.39-2.53)$ \\
\hline Father & $52 / 121$ & $1.69(1.16-2.44)$ & $1.63(1.11-2.37)$ & $162 / 121$ & $2.04(1.54-2.69)$ & $1.96(1.43-2.70)$ \\
\hline Both & $10 / 19$ & $1.59(0.69-3.66)$ & $1.53(0.66-3.54)$ & $53 / 19$ & $3.61(2.02-6.43)$ & $2.89(1.52-5.50)$ \\
\hline Any sibling & $74 / 96$ & $3.33(2.36-4.70)$ & $3.05(2.14-4.35)$ & $266 / 96$ & $3.18(2.44-4.15)$ & $2.90(2.15-3.90)$ \\
\hline Sister & $22 / 36$ & $2.71(1.54-4.77)$ & $2.39(1.34-4.26)$ & $86 / 36$ & $2.68(1.76-4.09)$ & $2.40(1.48-3.87)$ \\
\hline Brother & $38 / 49$ & $3.06(1.93-4.87)$ & $2.88(1.79-4.63)$ & $139 / 49$ & $3.12(2.18-4.47)$ & $2.92(1.95-4.36)$ \\
\hline Both & $8 / 7$ & $5.79(1.99-16.83)$ & $5.39(1.83-15.86)$ & $31 / 7$ & $5.23(2.16-12.71)$ & $4.83(1.86-12.53)$ \\
\hline Children & $13 / 28$ & $1.87(0.93-3.77)$ & $1.90(0.94-3.86)$ & $46 / 28$ & $1.83(1.08-3.10)$ & $1.28(0.71-2.30)$ \\
\hline Mother/sister & $59 / 150$ & $1.86(1.32-2.62)$ & $1.68(1.18-2.40)$ & $204 / 150$ & $2.42(1.89-3.11)$ & $2.14(1.60-2.85)$ \\
\hline Father/brother & $66 / 144$ & $2.30(1.65-3.20)$ & $2.17(1.55-3.05)$ & $220 / 144$ & $2.70(2.11-3.46)$ & $2.64(1.98-3.52)$ \\
\hline Both & $27 / 38$ & $3.66(2.17-6.19)$ & $3.28(1.92-5.61)$ & $130 / 38$ & $6.05(4.07-9.01)$ & $4.81(3.08-7.50)$ \\
\hline
\end{tabular}

a Model 1: adjusted for age, gender.

b Model 2: adjusted for age, gender, body mass index, education, smoking.

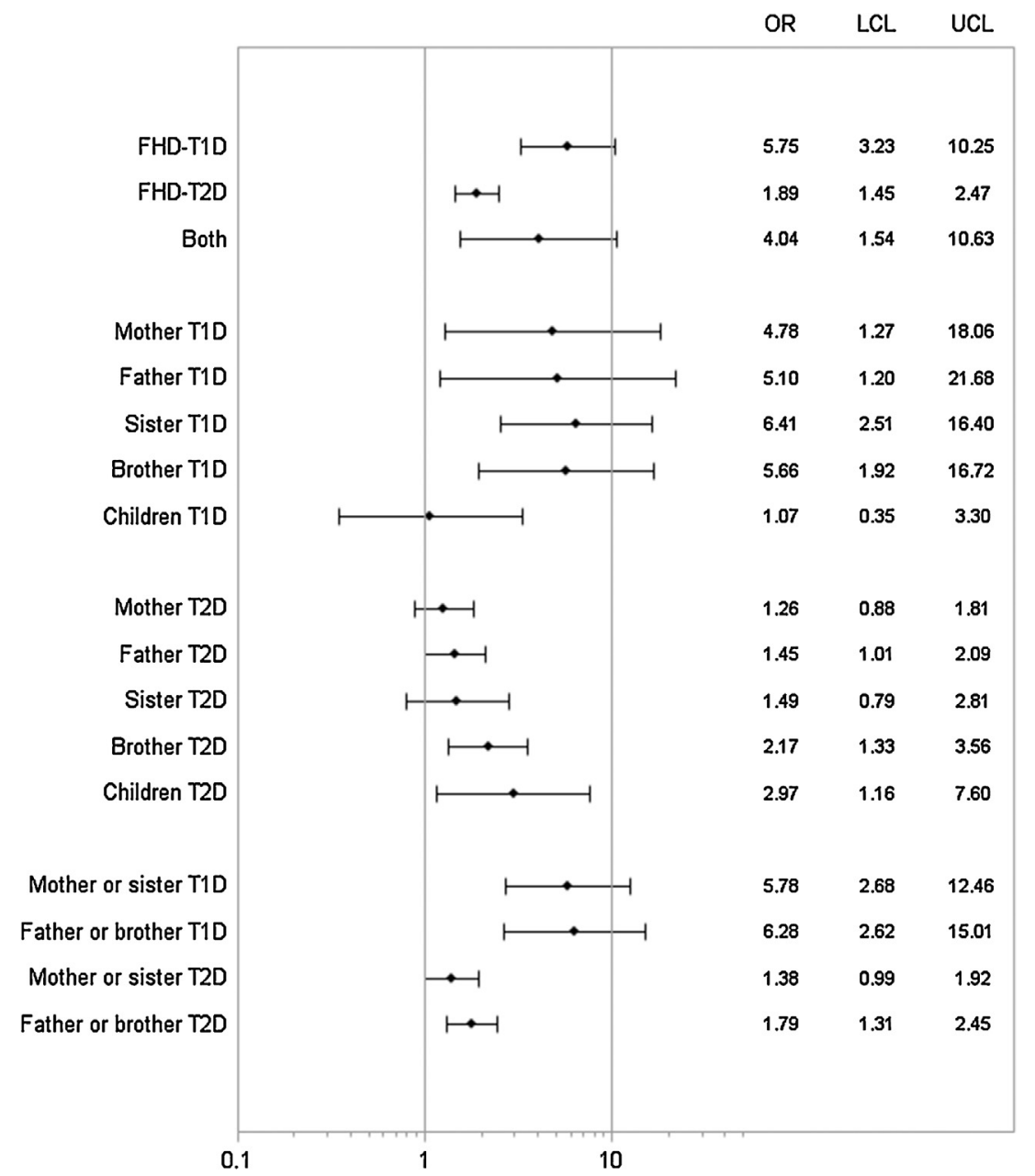

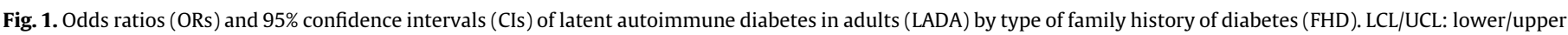
control limit; T1D/T2D: type 1/type 2 diabetes. 
Table 3

Characteristics of latent autoimmune diabetes in adults (LADA) by first-degree family history of diabetes (FHD).

\begin{tabular}{|c|c|c|c|c|c|c|}
\hline \multicolumn{7}{|l|}{ LADA } \\
\hline & No FHD & FHD & $P$ & FHD-T1D & FHD-T2D & $P$ \\
\hline Subjects, $n$ & 154 & $164^{\mathrm{a}}$ & & $30^{\mathrm{b}}$ & $126^{\mathrm{b}}$ & \\
\hline Women, \% & 37.0 & 54.9 & 0.0014 & 56.7 & 54.0 & 0.7897 \\
\hline Age at onset, years, mean (SD) & $59.7(12.6)$ & $59.4(12.1)$ & 0.8056 & $57.4(14.6)$ & $60.0(11.4)$ & 0.2932 \\
\hline $\mathrm{BMI}, \mathrm{kg} / \mathrm{m}^{2}$, mean $(\mathrm{SD})$ & $27.7(4.7)$ & $28.4(5.6)$ & 0.2218 & $27.9(6.0)$ & $28.8(5.4)$ & 0.4264 \\
\hline C-peptide, nmol/L, mean (SD) & $0.81(0.53)$ & $0.84(0.53)$ & 0.5866 & $0.65(0.43)$ & $0.91(0.54)$ & 0.0181 \\
\hline GADA, IU/mL, median (IQR) & $143.5(226.0)$ & $191.5(222.0)$ & 0.2697 & $250.0(169.0)$ & $104.0(226.0)$ & 0.0472 \\
\hline HOMA-IR, mean (SD) & $6.78(18.28)$ & $4.48(8.92)$ & 0.1973 & $3.25(2.83)$ & $4.32(8.88)$ & 0.5843 \\
\hline HOMA- $\beta$, mean (SD) & $45.43(36.42)$ & $46.66(34.32)$ & 0.7827 & $43.35(36.73)$ & $48.76(34.08)$ & 0.5119 \\
\hline HOMA-S, mean (SD) & $36.01(20.38)$ & $43.18(26.82)$ & 0.0197 & $51.38(34.15)$ & $41.18(24.24)$ & 0.1031 \\
\hline HLA DR4-DQ8, \% & 48.3 & 57.7 & 0.1573 & 70.0 & 53.6 & 0.1826 \\
\hline High-risk HLA genotypes ${ }^{c}, \%$ & 71.1 & 73.9 & 0.6358 & 90.0 & 69.1 & 0.0576 \\
\hline HLA $D R X / X, \%$ & 25.4 & 23.4 & 0.7251 & 5.0 & 28.6 & 0.0384 \\
\hline
\end{tabular}

IQR: interquartile range; HOMA-IR/ $\beta / S$ : homoeostasis model assessment of insulin resistance/ $\beta$-cell function/insulin sensitivity; HLA: human leucocyte antigen.

a Excluding $n=60$ with only second-degree FHD.

b Excluding $n=8$ with both FHD-T1D and FHD-T2D.

c $D R 4-D Q 8, D R 3 / 3, D R 4 / 4, D R 3 / 4, D R 3 / X, D R 4 / X$ combined.

linked to FHD-T2D (OR: 2.7, 95\% CI: 2.2-3.3), but not significantly to FHD-T1D (OR: 1.5, 95\% CI: 0.8-3.0) (Fig. S1; see supplementary materials associated with this article online). Compared with having a parent or child with T2D (Table SII; see supplementary materials associated with this article online), the excess risk appeared to be more pronounced in those whose siblings had T2D.

\section{Characteristics of patients by family history of diabetes}

LADA patients with FHD-T1D, compared with LADA patients with FHD-T2D, had lower levels of C-peptide and higher levels of GADA, lower prevalence of the low-risk genotype $D R X / X(5.0 \%$ vs $28.6 \%$, respectively; $P=0.0384)$, and a tendency for higher prevalences of the high-risk $D R 4-D Q 8$ (70.0\% vs 53.6\%, respectively; $P=0.1826)$ and the combined high-risk genotypes (90.0\% vs $69.1 \%$, respectively; $P=0.0576$ ) (Table 3 ). Only eight LADA patients had a mixed family history with both FHD-T1D and FHD-T2D. Compared with T2D patients, LADA patients with FHD-T2D had lower levels of C-peptide ( 0.91 vs $1.33 \mathrm{nmol} / \mathrm{L} ; P<0.0001)$ and HOMA- $\beta$ (48.76 vs 69.56, respectively; $P<0.0001$ ), and a lower frequency of the low-risk genotype ( $28.6 \%$ vs $47.8 \%$, respectively; $P=0.0008$ ) and a higher frequency of the high-risk genotype (53.6\% vs $26.3 \%$, respectively; $P<0.0001$ ). Characteristics of patients with T2D and FHD-T1D and FHD-T2D are presented in Table SIII (see supplementary materials associated with this article online). Only a few patients with T2D had exclusively FHD-T1D (2\%), and they had a lower prevalence of the low-risk genotype than those with FHDT2D, as well as a higher prevalence of the high-risk variants.

\section{Discussion}

Our present study is, to our knowledge, the first to explore the relative contributions of FHD-T1D and FHD-T2D to the risk of LADA. Indeed, our findings indicate that the risk of LADA is, by far, more pronounced in individuals with T1D in the family: a sixfold greater risk was associated with FHD-T1D in first-degree relatives compared with a twofold increased risk with FHD-T2D. Associations with both FHD-T1D and FHD-T2D lend support to the hypothesis of LADA as a genetic mix of T1D and T2D [10,11,32], although the strong association observed with FHD-T1D emphasizes the importance of genes related to autoimmunity in the promotion of LADA. Our present results also highlight the heterogeneity of LADA, demonstrating phenotype differences between patients with FHD-T1D and FHD-T2D. In contrast to previous findings in T1D $[1,33]$, the excess risk seems to be similar for female and male relatives. Furthermore, our present findings confirm previous observations that FHD is a strong risk factor for LADA $[12,13]$, and that the risk increases with the number and closeness of relatives. Adjusting for lifestyle factors explained only a small part of the excess risk associated with FHD, which is consistent with previous findings for T2D [2].

The heredity behind T1D is first and foremost transmitted by genes in the HLA class-II region [3], where the DR4-DQ8 haplotype is one of the strongest predictors [34]. As further support, LADA patients reporting FHD-T1D presented with lower prevalences of the protective $D R X / X$ genotype and tended to have higher prevalences of the high-risk HLA genotypes compared with LADA patients with FHD-T2D. Our findings have also indicated that FHD-T1D is associated with a more T1D-like LADA, with more autoimmunity. This fits with previous reports that the degree of autoimmunity in LADA is linked to the frequency of HLA DQB1 genotypes [6,7,32]. Although the genes linked to T2D were not studied, our findings are consistent with a link between genes related to T2D and LADA, as shown elsewhere [9-11]. Another explanation is that shared lifestyle factors promoting T2D may also be associated with LADA. The few previous studies of a family history of T1D vs T2D in LADA support our present findings; Fourlanos et al. [20] found that FHD-T2D is almost as common in LADA patients as in T2D patients, whereas FHD-T1D is significantly more prevalent. Studies of T2D are also in keeping with our data by suggesting that a family history of both types are more common in T2D patients positive for GAD autoantibodies than in GADAnegative patients [16-18].

The risk of T2D was primarily linked to FHD-T2D. Patients with FHD had lower levels of C-peptide than those without FHD, which is consistent with previous studies $[12,35]$. In line with this, a majority of the most common gene variants for T2D, including variants of the TCF7L2 gene [4], have been suggested to affect disease development via impaired insulin secretion rather than insulin resistance $[5,35]$. On the other hand, no differences were found in levels of HOMA- $\beta$ between the two groups, suggesting that this interpretation of our study results should be made with caution. Also, the association between T2D and FHD-T1D was weak. One implication of this finding is that, for subjects who present with T2D-like symptoms but report FHD-T1D, it may be advisable to screen for GADA, as these patients may have LADA. 


\section{Strengths and limitations}

Our study strengths include the large number of LADA cases, with detailed information on FHD and potential confounders, as well as the population-based nature of the study. The main limitation was that the information on FHD was self-reported. Also, patients with diabetes may be more likely to remember or enquire about their relatives with diabetes, and relatives may also be more inclined to seek medical care for symptoms, which may have led to overestimation of the association between FHD and diabetes. On the other hand, as all patients were newly diagnosed, the period of increased awareness among relatives was brief.

It should also be noted that the type of diabetes in relatives was classified based on age at onset and use of insulin treatment as recalled by the participant. Importantly, individuals classified as having FHD-T1D were more likely to have the $D R 4-D Q 8$ genotype associated with T1D, whereas FHD-T2D was associated with the low-risk $D R X / X$ genotype, supporting the validity of self-reporting. Still, some relatives classified as having T2D may, in fact, have had autoimmune diabetes with onset at age $>40$. This may have contributed to an association between LADA and FHD-T2D. However, after re-running the analysis with a stricter FHD-T2D classification ( $\geq 40$ years and no insulin treatment), an association with LADA was still observed. False-positive cases of LADA might also explain the association with FHD-T2D, although this seems unlikely, as the association with FHD-T2D was found even when the analysis was restricted to LADA patients with high (above median) GADA levels. Moreover, LADA patients with FHD-T2D differed from patients with T2D in both clinical and genetic characteristics.

The sensitivity of the GADA assay implies that some patients with LADA were classified as GADA-negative (having T2D). Also, even though it has been shown that GADA is by far the most common antibody in LADA, present in around $90 \%$ of all patients [36], other unmeasured autoantibodies, such as insulinomaassociated protein 2 and zinc transporter-8, may have been present. This would explain the tendency for the observed association between FHD-T1D and T2D. Notably, T2D patients with FHD-T1D had a lower prevalence of the low-risk HLA genotypes and a higher prevalence of the high-risk HLA genotypes than those with FHD-T2D. Finally, it should be noted that the incidence of T1D in Scandinavia is the highest in the world [37], and the relative importance of FHD-T1D and FHD-T2D may differ from those of populations with lower incidences and different genetic bases.

In conclusion, having both T1D and T2D in the family is associated with the risk of LADA, and the strong association with FHD-T1D suggests that genes related to autoimmunity are more influential in the development of LADA than those related to T2D. Given this situation, it is noteworthy that, with regard to lifestyle, studies to date indicate that LADA shares several risk factors with T2D, including smoking [38], alcohol consumption (protective) [39] and low birth weight [40]. Taken together, these findings indicate that LADA development is promoted by genes related to autoimmunity and by lifestyle factors that increase insulin resistance.

\section{Author contributions}

S.C., P.-O.C., L.G., M.M., B.R. and P.S. contributed to the collection of data, interpretation of the results and writing of the manuscript. L.A., V.G. and T.T. interpreted the results and participated in the writing and editing of the manuscript. T.A. contributed with statistical considerations, analysing the data and writing of the manuscript. S.C. conceptualized the research objectives and designed the study. R.H. contributed to the conduct of the study and was responsible for analysing the data and writing the paper. R.H. and S.C. are the guarantors of this work and takes responsibility for the integrity of the data and the accurateness of the data analysis. All authors critically revised and approved the final version of the manuscript.

\section{Funding}

The ESTRID study was funded by grants from the Swedish Medical Research Council, the Swedish Research Council for Health, Working life and Welfare, AFA Insurance Company, the Swedish Diabetes Association and the Novo Nordisk Foundation. ANDIS was funded by grants from the Swedish Medical Research Council and ERC Advanced Researcher grant (GA 269045) to LG and ALF- Swedish Research Council funding for Clinical research. Funding for ANDIU was provided by the Swedish Medical Research Council, a strategic Research Grant from the Swedish Government (excellence of diabetes research in Sweden-EXODIAB).

\section{Disclosure of interest}

The authors declare that they have no competing interest.

\section{Appendix A. Supplementary data}

Supplementary materials (Fig. S1, and Tables SI-SIII) associated with this article can be found at http://www.scincedirect.com at http://dx.doi.org/10.1016/j.diabet.2017.05.010.

\section{References}

[1] Weires MB, Tausch B, Haug PJ, Edwards CQ, Wetter T, Cannon-Albright LA. Familiality of diabetes mellitus. Exp Clin Endocrinol Diabetes 2007;115:63440.

[2] InterAct $C$, Scott RA, Langenberg $C$, Sharp SJ, Franks PW, Rolandsson 0 , et al The link between family history and risk of type 2 diabetes is not explained by anthropometric, lifestyle or genetic risk factors: the EPIC-InterAct study. Diabetologia 2013;56:60-9.

[3] Noble JA, Valdes AM. Genetics of the HLA region in the prediction of type 1 diabetes. Curr Diab Rep 2011;11:533-42.

[4] Grant SF, Thorleifsson G, Reynisdottir I, Benediktsson R, Manolescu A, Sainz J, et al. Variant of transcription factor 7-like 2 (TCF7L2) gene confers risk of type 2 diabetes. Nat Genet 2006;38:320-3.

[5] Prasad RB, Groop L. Genetics of type 2 diabetes-pitfalls and possibilities. Genes (Basel) 2015;6:87-123

[6] Tuomi T, Santoro N, Caprio S, Cai M, Weng J, Groop L. The many faces of diabetes: a disease with increasing heterogeneity. Lancet 2014;383:1084-94.

[7] Andersen MK, Lundgren V, Turunen JA, Forsblom C, Isomaa B, Groop PH, et al. Latent autoimmune diabetes in adults differs genetically from classical type 1 diabetes diagnosed after the age of 35 years. Diabetes Care 2010;33:2062-4.

[8] Laugesen E, Ostergaard JA, Leslie RD, Danish Diabetes Academy W, Workshop S. Latent autoimmune diabetes of the adult: current knowledge and uncertainty. Diabet Med 2015.

[9] Lukacs K, Hosszufalusi N, Dinya E, Bakacs M, Madacsy L, Panczel P. The type 2 diabetes-associated variant in TCF7L2 is associated with latent autoimmune diabetes in adult Europeans and the gene effect is modified by obesity: a metaanalysis and an individual study. Diabetologia 2012;55:689-93.

[10] Cervin C, Lyssenko V, Bakhtadze E, Lindholm E, Nilsson P, Tuomi T, et al. Genetic similarities between latent autoimmune diabetes in adults, type 1 diabetes, and type 2 diabetes. Diabetes 2008;57:1433-7.

[11] Andersen MK, Sterner M, Forsen T, Karajamaki A, Rolandsson O, Forsblom C, et al. Type 2 diabetes susceptibility gene variants predispose to adult-onset autoimmune diabetes. Diabetologia 2014:57:1859-68.

[12] Carlsson S, Midthjell K, Grill V. Influence of family history of diabetes on incidence and prevalence of latent autoimmune diabetes of the adult: results from the Nord-Trondelag Health Study. Diabetes Care 2007;30:3040-5.

[13] Lundgren VM, Isomaa B, Lyssenko V, Laurila E, Korhonen P, Groop LC, et al. GAD antibody positivity predicts type 2 diabetes in an adult population. Diabetes 2010;59:416-22.

[14] Zhou Z, Xiang Y, Ji L, Jia W, Ning G, Huang G, et al. Frequency, immunogenetics, and clinical characteristics of latent autoimmune diabetes in China (LADA China study): a nationwide, multicenter, clinic-based cross-sectional study. Diabetes 2013;62:543-50. 
[15] Schloot NC, Pham MN, Hawa MI, Pozzilli P, Scherbaum WA, Schott M, et al. Inverse relationship between organ-specific autoantibodies and systemic immune mediators in type 1 diabetes and type 2 diabetes: action LADA 11. Diabetes Care 2016;39:1932-9.

[16] Li H, Isomaa B, Taskinen MR, Groop L, Tuomi T. Consequences of a family history of type 1 and type 2 diabetes on the phenotype of patients with type 2 diabetes. Diabetes Care 2000;23:589-94.

[17] Li H, Lindholm E, Almgren P, Gustafsson A, Forsblom C, Groop L, et al. Possible human leukocyte antigen-mediated genetic interaction between type 1 and type 2 Diabetes. J Clin Endocrinol Metab 2001;86:574-82.

[18] Lundgren VM, Andersen MK, Isomaa B, Tuomi T. Family history of Type 1 diabetes affects insulin secretion in patients with 'Type 2' diabetes. Diabet Med 2013;30:e163-9.

[19] Castleden HA, Shields B, Bingley PJ, Williams AJ, Sampson M, Walker M, et al. GAD antibodies in probands and their relatives in a cohort clinically selected for type 2 diabetes. Diabet Med 2006;23:834-8.

[20] Fourlanos S, Perry C, Stein MS, Stankovich J, Harrison LC, Colman PG. A clinical screening tool identifies autoimmune diabetes in adults. Diabetes Care 2006;29:970-5.

[21] Lofvenborg JE, Andersson T, Carlsson PO, Dorkhan M, Groop L, Martinell M, et al. Coffee consumption and the risk of latent autoimmune diabetes in adults - results from a Swedish case-control study. Diabet Med 2014;31:799-805.

[22] Lund University. All New Diabetics in Scania, http://www.andis.ludc.med.lu.se [accessed 16.12.28].

[23] Vandenbroucke JP, Pearce N. Case-control studies: basic concepts. Int J Epidemiol 2012;41:1480-9.

[24] Uppsala University. All new diabetics in Uppsala County, http://www.andiu. se/ [accessed 16.12.28].

[25] Rahmati K, Lernmark A, Becker C, Foltyn-Zadura A, Larsson K, Ivarsson SA, et al. A comparison of serum and EDTA plasma in the measurement of glutamic acid decarboxylase autoantibodies (GADA) and autoantibodies to islet antigen-2 (IA-2A) using the RSR radioimmunoassay (RIA) and enzyme linked immunosorbent assay (ELISA) kits. Clin Lab 2008;54:227-35.

[26] Smolcic VS, Bilic-Zulle L, Fisic E. Validation of methods performance for routine biochemistry analytes at Cobas 6000 analyzer series module c501. Biochem Med (Zagreb) 2011;21:182-90.

[27] Brophy S, Yderstraede K, Mauricio D, Hunter S, Hawa M, Pozzilli P, et al. Time to insulin initiation cannot be used in defining latent autoimmune diabetes in adults. Diabetes Care 2008;31:439-41.
[28] The Oxford Center for Diabetes. Endocrinology \& Metabolism. Diabetes Trial Unit. HOMA Calculator, http://www.dtu.ox.ac.uk/homacalculator [accessed 16.02.01]

[29] Nguyen C, Varney MD, Harrison LC, Morahan G. Definition of high-risk type 1 diabetes HLA-DR and HLA-DQ types using only three single nucleotide polymorphisms. Diabetes 2013;62:2135-40.

[30] Erlich H, Valdes AM, Noble J, Carlson JA, Varney M, Concannon P, et al HLA DR-DQ haplotypes and genotypes and type 1 diabetes risk: analysis of the type 1 diabetes genetics consortium families. Diabetes 2008;57:1084-92.

[31] Sepp H, Ekelund U, Becker W. Enkätfrågor om kost och fysisk aktivitet bland vuxna. Livsmedelverket, rapport 21; 2004.

[32] Pettersen E, Skorpen F, Kvaloy K, Midthjell K, Grill V. Genetic heterogeneity in latent autoimmune diabetes is linked to various degrees of autoimmune activity: results from the Nord-Trondelag Health Study. Diabetes 2010:59:302-10

[33] Gale EA, Gillespie KM. Diabetes and gender. Diabetologia 2001;44:3-15.

[34] Pociot F, Lernmark A. Genetic risk factors for type 1 diabetes. Lancet 2016;387:2331-9.

[35] Lyssenko V, Jonsson A, Almgren P, Pulizzi N, Isomaa B, Tuomi T, et al. Clinical risk factors, DNA variants, and the development of type 2 diabetes. N Engl J Med 2008;359:2220-32.

[36] Hawa MI, Kolb H, Schloot N, Beyan H, Paschou SA, Buzzetti R, et al. Adult-onset autoimmune diabetes in Europe is prevalent with a broad clinical phenotype: action LADA 7. Diabetes Care 2013;36:908-13.

[37] Patterson C, Guariguata L, Dahlquist G, Soltesz G, Ogle G, Silink M. Diabetes in the young - a global view and worldwide estimates of numbers of children with type 1 diabetes. Diabetes Res Clin Pract 2014;103:161-75.

[38] Rasouli B, Andersson T, Carlsson PO, Grill V, Groop L, Martinell M, et al. Smoking and the risk of LADA: results from a Swedish population-based case-control study. Diabetes Care 2016;39:794-800.

[39] Rasouli B, Andersson T, Carlsson PO, Dorkhan M, Grill V, Groop L, et al. Alcohol and the risk for latent autoimmune diabetes in adults: results based on Swedish ESTRID study. Eur J Endocrinol 2014;171:535-43.

[40] Hjort R, Alfredsson L, Carlsson PO, Groop L, Martinell M, Storm P, et al. Low birthweight is associated with an increased risk of LADA and type 2 diabetes: results from a Swedish case-control study. Diabetologia 2015;58:2525-32. 